\section{Polarité planaire et polykystose rénale}

Evelyne Fischer, Marco Pontoglio
Expression génétique et Maladies,

FRE 2850 CNRS, Institut Pasteur,

25, rue du Docteur Roux,

75724 Paris Cedex 15, France.

marcop@pasteur.fr

> La polykystose rénale est une maladie génétique très fréquente (1/1000) qui pose un problème majeur de santé publique. En effet, $50 \%$ des patients atteints de cette pathologie doivent avoir recours à un traitement par dialyse ou transplantation rénale au cours de leur vie. Malgré l'importance de cette pathologie, les mécanismes cellulaires et moléculaires impliqués dans les étapes initiales de la polykystose (dilatation tubulaire) restent encore mal connus. Des études récentes ont permis d'élucider certaines de ces étapes précoces. Un modèle murin de polykystose lié à l'inactivation du gène TCF2/HNFI $\beta$ dans le rein a été particulièrement utile. HNFl $\beta$ est un facteur de transcription dimérique à homéodomaine atypique, exprimé dans les cellules épithéliales du rein, du foie, du pancréas, et du poumon. Dans le rein, HNFl $\beta$ est exprimé très tôt au cours du développement, dans le mésonephros, puis dans le bourgeon urétéral et ses dérivés, le système collecteur, ainsi que dans les tubules rénaux dérivant du blastème métanéphrique [1].

Chez l'homme, des mutations dans ce gène sont responsables d'anomalies du développement rénal (dysplasie) mais aussi d'anomalies tubulaires plus tardives (kystes). Chez la souris, l'inactivation germinale d'HNFI $\beta$ est létale au cours du développement (દ6.5-દ7.5) à la suite d'un défaut de différenciation des annexes extraembryonnaires [2, 3]. L'inactivation conditionnelle de ce gène, spécifiquement dans le rein, conduit à une polykystose due à une diminution drastique de l'expression des gènes Pkhdl et Pkd2 [4], dont les mutations sont connues pour être responsable de 


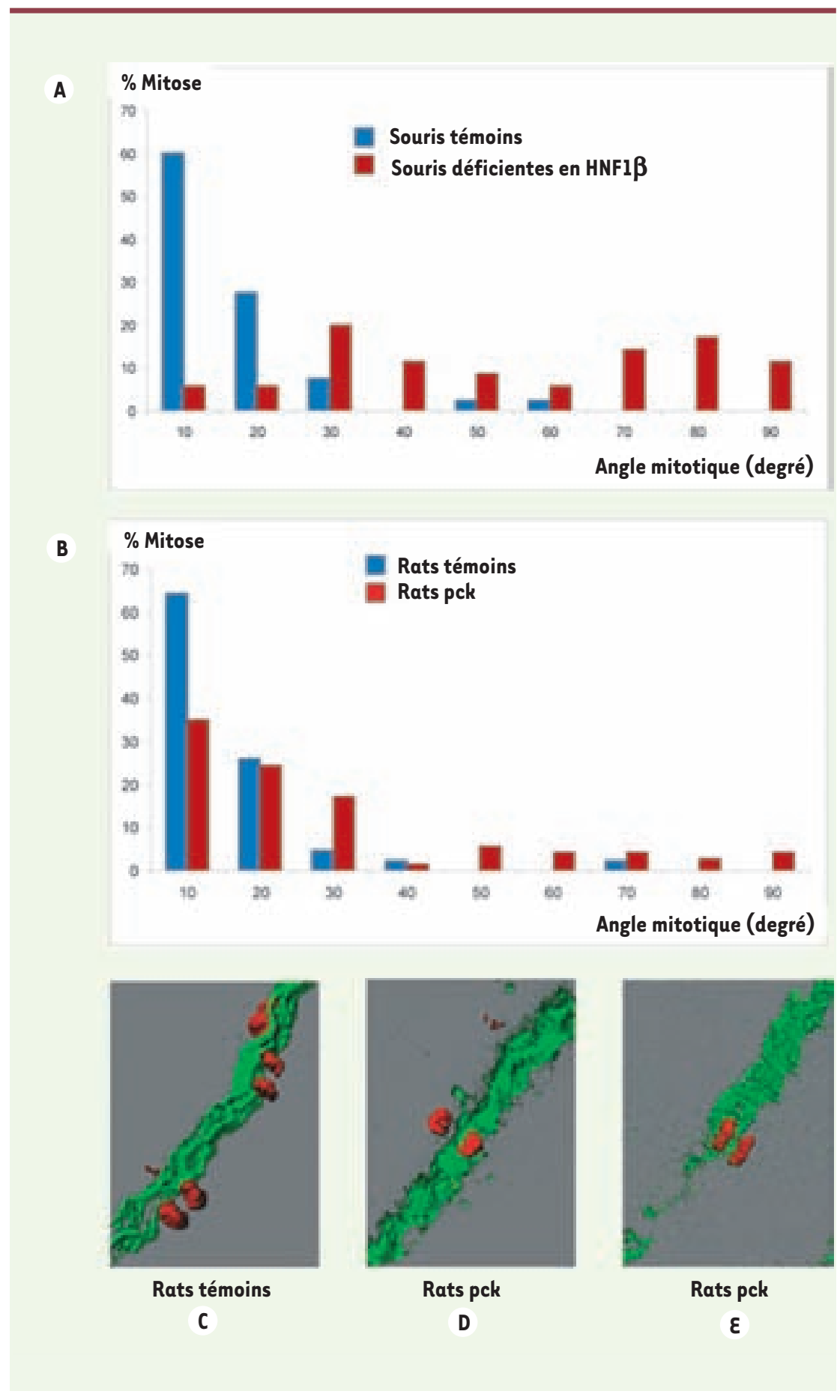

Figure 2. Orientation de la mitose dans les cellules tubulaires. A. Comparaison de la distribution de l'angle mitotique (angle entre l'axe du tube et l'axe du fuseau mitotique) entre souris sauvages et souris déficientes en HNFl $\beta$ dans le rein. Chez ces souris polykystiques, l'angle mitotique est distribué de façon aléatoire, alors qu'il est orienté selon l'axe du tube chez les souris témoins. B. Comparaison de la distribution de l'angle mitotique chez des rats témoins et des rats pck. Ces mesures ont été effectuées avant l'apparition des kystes, dans des tubes dont le calibre est comparable à celui des rats témoins. Déjà, à ce stade, une proportion significative des mitoses n'est plus orientée selon l'axe du tube chez les rats pck. Un exemple de mitoses alignées longitudinalement chez le rat témoin est représenté en $C$. Des mitoses orientées de façon aberrante chez le rat pck sont montrées en $\boldsymbol{D}$ et en $\boldsymbol{\varepsilon}$ (d'après [8]) pathologies polykystiques (pour revue, voir [5]). Le contrôle transcriptionnel par HNFl $\beta$ de ces gènes pourrait expliquer la formation de kystes chez les patients porteurs de mutations dans HNFI $\beta$. Pendant la phase de maturation des néphrons, période pendant laquelle apparaissent les kystes dans les modèles d'inactivation de gènes de polykystose $(P k d l$ et $P k d 2)[6,7]$, les tubules s'allongent de façon considérable en raison d'une intense prolifération cellulaire. De façon remarquable, cette prolifération allonge les tubules sans augmenter leur diamètre. Cette «propriété » (allongement sans dilatation) pourrait être la conséquence d'un contrôle de l'orientation de la division cellulaire selon l'axe du tubule [8] (Figure 1, partie 1). Cette hypothèse a été testée en utilisant des souris transgéniques porteuses d'une cassette $\beta$-galactosidase (souris ROSA26R) [9] dont l'expression est activable par l'action d'une Cre recombinase inductible par le tamoxifène [10]. Chez ces animaux, des doses suboptimales de cette drogue induisent, dans un nombre limité de cellules tubulaires, l'expression de la $\beta$-galactosidase qui persiste et se transmet de façon stable dans toutes les cellules filles. La forme des territoires occupés par les cellules exprimant la $\beta$-galactosidase (analyse clonale rétrospective) a montré que les cellules filles, issues des divisions successives d'une cellule tubulaire, restent en contact les unes avec les autres et sont alignées le long du tubule (Figure 1, partie $2 A-D$ ). Dans une autre série d'expériences, la mesure de l'angle mitotique (angle entre l'axe du tube et l'axe du fuseau mitotique) a montré que l'alignement des cellules filles est dû à une stricte orientation des mitoses selon l'axe du tubule. Cette orientation de la division cellulaire devrait permettre aux tubules de s'allonger en conservant un diamètre constant.

La polykystose rénale, en particulier dans sa forme autosomique récessive, est caractérisée par une augmentation progressive du diamètre tubulaire aboutissant 
à la formation de kystes (pour revue, voir [11]). Dans le modèle murin de polykystose par inactivation de HNFI $\beta$ dans le rein [4], la dilatation tubulaire est associée à une augmentation du nombre de cellules par section tubulaire. Remarquablement, chez ces souris, l'angle mitotique, pendant la phase d'allongement tubulaire, est distribué de façon aléatoire (Figure 2A). Afin de vérifier si la désorganisation des mitoses est à l'origine de la dilation tubulaire, et non secondaire à la présence de kystes, l'orientation des mitoses a été analysée dans un second modèle animal, le rat pck [12]. Dans ce modèle, la formation des kystes, liée à une importante diminution de l'expression de Pkhdl, débute plus tardivement que chez les souris déficientes en HNFl $\beta$ dans le rein. Déjà, à un stade précédant la dilatation tubulaire, un nombre significatif de mitoses n'est plus orienté selon l'axe du tube (Figure 2, $B, C-\varepsilon$ ). Ces résultats suggèrent une relation de cause à effet entre la perte de l'orientation de la division cellulaire et la dilatation tubulaire (polykystose) observées chez ces modèles animaux.

L'orientation de la division cellulaire selon l'axe tubulaire est une manifestation de polarité planaire qui oriente vectoriellement la cellule dans le plan de l'épithélium. Les cellules épithéliales tubulaires dans le rein présentent donc, outre une polarité apicobasale, une polarité dont l'axe est orienté perpendiculairement à l'axe apico-basal.

Cette polarisation cellulaire planaire (PCP) régule les mouvements cellulaires au cours de la morphogenèse (convergence-extension) et l'orientation des cellules au sein des tissus (pour revue, voir [13]). L'interaction directe entre des cystoprotéines, localisées dans le cil primaire, et les acteurs de la voie de signalisation de la polarité planaire a été illustrée par des études récentes. L'une de ces études décrit l'interaction entre inversine, une protéine dont les mutations sont responsables de la néphronophthise de type 2 , et dishevelled, protéine clé de la voie wnt canonique et impliquée aussi dans la signalisation PCP [14]. Un autre travail montre une colocalisation cellulaire de l'une des protéines responsable du syndrome de Bardet-Biedl (incluant aussi une pathologie kystique), BBS6/Mkks, et strabismus, l'une des protéines impliquées dans la polarisation planaire [15]. De plus, Mks3, un gène récemment identifié dans le syndrome de Meckel-Gruber, syndrome comprenant une anomalie kystique rénale, présente une similarité topologique avec les protéines de la famille frizzled, récepteurs impliqués dans la signalisation PCP [16]. L'ensemble des résultats décrits ci-dessus suggère que les cellules rénales tubulaires sont polarisées dans le plan de l'épithélium, et que le cil primaire pourrait participer à cette polarisation. Cet organite est considéré comme un dispositif mécanosenseur du flux urinaire (pour revue, voir $[5,16])$. II est tentant de spéculer que le flux urinaire pourrait fournir une orientation vectorielle qui permettrait une organisation géométrique polarisée dans le plan de la cellule tubulaire rénale. En interrompant la signalisation via les cystoprotéines du cil primaire, la polarité planaire pourrait être altérée et provoquer la dilatation tubulaire observée dans les modèles de polykystose. $\diamond$
Planar cell polarity

and polycystic kidney disease

\section{RéFÉRENCES}

1. Coffinier C, Barra J, Babinet C, et al. Expression of the vHNFl/HNFlbeta homeoprotein gene during mouse organogenesis. Mech Dev 1999; 89 : 211-3.

2. Coffinier C, Thepot D, Babinet C, et al. Essential role for the homeoprotein vHNFI/HNFl beta in visceral endoderm differentiation. Development 1999; 126 : 4785-94.

3. Barbacci $\varepsilon$, Reber M, Ott MO, et al. Variant hepatocyte nuclear factor $l$ is required for visceral endoderm specification. Development $1999 ; 126$ : 4795-805.

4. Gresh L, Fischer $\varepsilon$, Reimann A, et al. A transcriptional network in polycystic kidney disease. EMBO J 2004 ; $23: 1657-68$

5. Igarashi P, Somlo S. Genetics and pathogenesis of polycystic kidney disease. J Am Soc Nephrol 2002; 13: 2384-98.

6. Wu G, D'Agati V, Cai Y, et al. Somatic inactivation of Pkd2 results in polycystic kidney disease. Cell 1998; $93: 177-88$.

7. Lu W, Peissel B, Babakhanlou H, et al. Perinatal lethality with kidney and pancreas defects in mice with a targetted Pkdl mutation. Nat Genet 1997 ; 17 : 179-81.

8. Fischer $\varepsilon$, Legue $\varepsilon$, Doyen A, et al. Defective planar cell polarity in polycystic kidney disease. Nat Genet 2006 ; 38: $21-3$.

9. Soriano P. Generalized lacZ expression with the ROSA26 Cre reporter strain. Nat Genet 1999; $21: 70-1$.

10. Vooijs M, Jonkers J, Berns A. A highly efficient ligandregulated Cre recombinase mouse line shows that LoxP recombination is position dependent. EMBO Rep 2001; $2: 292-7$.

11. Wilson PD. Polycystic kidney disease : new understanding in the pathogenesis. Int J Biochem Cell Biol 2004 ; 36: 1868-73.

12. Lager DJ, (ian Q, Bengal RJ, et al. The pck rat : a new model that resembles human autosomal dominant polycystic kidney and liver disease. Kidney Int 2001 ; 5 : 126-36.

13. Axelrod JD, McNeill H. Coupling planar cell polarity signaling to morphogenesis. Scientific World J 2002 ; 2 : 434-54.

14. Simons M, Gloy J, Ganner A, et al. Inversin, the gene product mutated in nephronophthisis type II, functions as a molecular switch between Wnt signaling pathways. Nat Genet 2005 ; 37 : 537-43.

15. Ross AJ, May-Simera H, Eichers ER, et al. Disruption of Bardet-Biedl syndrome ciliary proteins perturbs planar cell polarity in vertebrates. Nat Genet 2005; 37 : 1135-40.

16. Smith UM, Consugar M, Tee LJ, et al. The transmembrane protein meckelin (MKS3) is mutated in Meckel-Gruber syndrome and the wpk rat. Nat Genet 2006 ; 38 : 191-6.

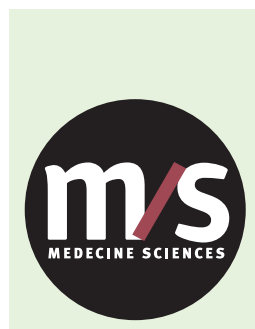
Tarifs d'abonnement M/S - 2006
> Depuis 20 ans, grâce à $\mathrm{m} / \mathrm{s}$, vous vivez en direct les progrès des sciences biologiques et médicales

Abonnez-vous

à Médecine/Sciences

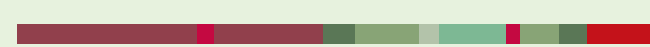

Bulletin d'abonnement page 564 dans ce numéro de $\mathrm{m} / \mathrm{s}$
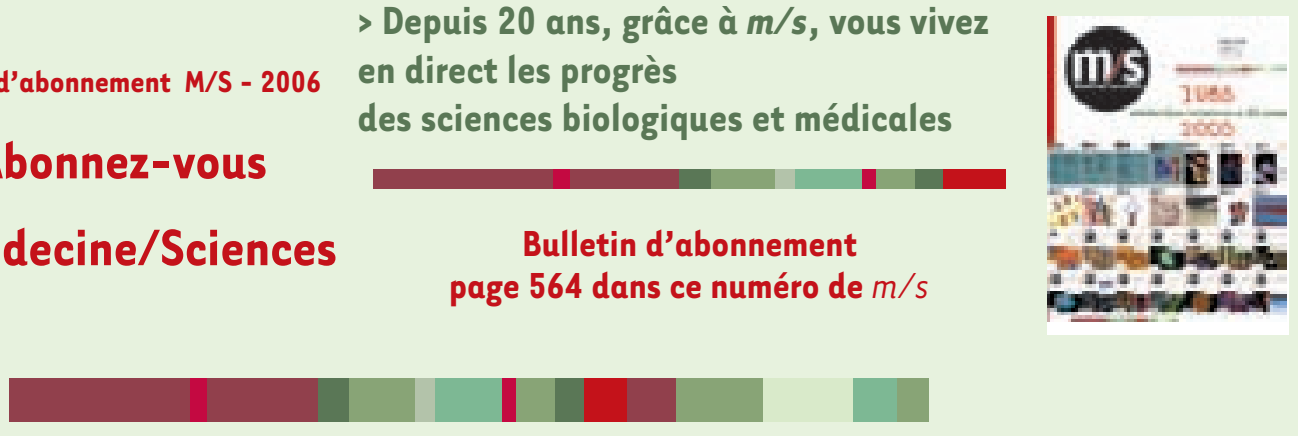